\title{
Neutral and charged electron-hole complexes in artificial molecules: Quantum transitions induced by the in-plane magnetic field
}

\author{
Devis Bellucci,* Filippo Troiani, Guido Goldoni, and Elisa Molinari \\ INFM-National Research Center on Nano-Structures and Bio-Systems at Surfaces $\left(S^{3}\right)$ and Dipartimento di Fisica, \\ Università degli Studi di Modena e Reggio Emilia, Via Campi 213/A, 41100 Modena, Italy
}

(Received 7 July 2004; published 22 November 2004)

\begin{abstract}
We theoretically investigate the properties of neutral and charged excitons and of the biexciton in vertically coupled quantum dots, as a function of the in-plane magnetic field $B_{\|}$. The main effect of the field consists in the suppression of the bonding-antibonding splitting, and in the resulting enhancement of the interdot correlations. As a consequence, the excitons form with the additional carrier a bound or an unbound complex depending on the sign of the charging, whereas the biexciton undergoes a transition between different quantum states with increasing $B_{\|}$. The discussed behaviors and transitions show up in the field dependence of experimentally accessible quantities, such as the charged-exciton and biexciton binding energies.
\end{abstract}

DOI: 10.1103/PhysRevB.70.205332

PACS number(s): 73.21.La, 03.67.-a, 71.35.-y

\section{INTRODUCTION}

The complete spatial confinement of the carriers achieved in semiconductor quantum dots (QDs) results in novel physical phenomena of fundamental interest ${ }^{1}$ and makes them potential building blocks of future quantum-devices and computers. ${ }^{2}$ Indeed, the continuous refinement of the different fabrication techniques achieved in the last two decades has allowed an increasing degree of control on the geometry, composition and spatial arrangement of these "artificial atoms," as well as a fine engineering of their electronic and optical properties. ${ }^{3}$

Particularly relevant in this last respect and in view of possible optoelectronic applications are the self-assembled quantum dots (SADs). Typical SADs are quasi-twodimensional (2D) systems, with an in-plane spatial confinement much weaker than the one in the growth $(z)$ direction. ${ }^{4}$ In recent years the formation of QD pillars with tuneable interdot barriers has also been demonstrated, where the interdot tunneling of the carriers results in a coherent coupling between neighboring dots and in the delocalization of the wave functions over the so-called "artificial molecule." ${ }^{5}$ As a consequence, the orbital excitations associated to the motion along $z$, which are effectively frozen in single QDs and in planar artificial molecules, become energetically accessible to applied electric and magnetic fields, thus giving the system a full three-dimensional character.

Detailed information on the interband excitations of artificial atoms are provided by their optical response and by its dependence on the applied magnetic field. Typical features emerging in the magneto-optical studies of electrically neutral $^{6}$ and charged ${ }^{7}$ QDs are confinement-regime dependent diamagnetic shifts of the exciton energies, removal of the degeneracy characterizing cylindrically symmetric dots, estimates of the electron-hole (reduced) effective mass and of the carrier separation. More recently, the effect of a vertical magnetic field on the optical and transport properties of coupled dots has also been proposed as a sensitive test of the interdot coherent coupling ${ }^{8}$ and of the few-electron quantum phase, ${ }^{9}$ respectively. Indeed, a magnetic field is a precious tool for the investigation of low-dimensional heterostructures, specially those characterized by length scales comparable to that of the magnetic confinement. Therefore, while in single, nearly 2D QDs the only relevant component of the field is the one along the growth direction $\left(B_{\perp}\right)$, in vertically coupled dots the in-plane component $\left(B_{\|}\right)$ also plays a crucial role: On the one hand, it breaks the system's possible cylindrical symmetry and mixes the degrees of freedom corresponding to the in-plane and vertical motion; on the other hand, it tunes the interdotcoupling regime, thus inducing in the few-particle states transitions reminescent of those that one can in principle obtain by varying the width of the interdot barrier. ${ }^{10}$ Due to its relevance to the QD-based implementation of quantuminformation processing ${ }^{11}$ such transverse-field induced transitions have been already investigated in the two-electron case, ${ }^{12}$ whereas less attention has been devoted to that of electron-hole complexes.

In this paper we theoretically investigate the field dependence of neutral and charged excitons, and of biexcitons in vertically coupled QDs. The main focus is on the interdotcorrelation effects, largely enhanced by the in-plane magnetic field $B_{\|}$and essentially determining carrier localization within the artificial molecules. In fact, whereas in semiconductor heterostructures of higher dimensionality the formation of electron-hole complexes is due to genuine Coulomb correlations, in artificial atoms it mainly arises from the three-dimensional (3D) spatial confinement of the carriers. In particular, in the strong-confinement limit the Coulomb effects only provide energy renormalizations, which can be qualitatively described within a perturbative picture. The artificial molecules of our present concern are somehow in between these two limiting cases. In fact, while strongly confined in the plane, the carriers can still localize in two different sites (dots) in the growth direction. As a result, the bound or unbound nature of the few-particle states is determined by the combined effect of the intradot (confinementinduced) Coulomb interactions and of the interdot spatial correlations.

The paper is organized as follows: In Sec. II we illustrate our numerical approach to the calculation of the single- and 
few-particle states, as well as to the optical and correlation properties of the latter; in Sec. III we discuss the results, concerning single electrons and holes, neutral- and chargedexcitons, and biexcitons; in Sec. IV we finally summarize our findings.

\section{THEORETICAL MODEL}

As a first step, we calculate the electron and hole singleparticle (SP) states, as determined by the 3D confinement and by the applied magnetic field (Sec. II A). These results provide the ingredients for the investigation of the Coulombinteracting electron-hole complexes (Sec. II B). Finally, we compute the carrier-carrier pair-correlation functions, further characterizing the few-particle states, and the optical properties of the system, resulting from the radiative recombination of electron-hole pairs (Sec. II C).

\section{A. Single-particle states}

Our modelling of the double-dot SP properties is developed within the theoretical framework of the envelopefunction approximation, which is known to give a good description of the system, provided that the confining potential (i.e., the heterostructure composition) varies sufficiently slowly on the length scale of the lattice constant. ${ }^{1}$ Besides, since the energy region of our concern is relatively close to the semiconductor band gap, we use the single-band approximation for both the conduction and the valence bands (effective-mass approach). The SP energies $\epsilon_{\alpha}^{\chi}$ and wave function $\phi_{\alpha}^{X}(\mathbf{r})$ thus correspond to the eigenvalues and eigenstates of the following Hamiltonian:

$$
h_{\mathrm{orb}}^{\chi}(\mathbf{r})=\frac{1}{2 m_{\chi}^{*}}\left(-i \hbar \nabla \pm \frac{|e|}{c} \mathbf{A}\right)^{2}+V^{\chi}(\mathbf{r}),
$$

where the vector potential is $\mathbf{A}=\frac{1}{2} \mathbf{B} \times \mathbf{r}$ (symmetric gauge), while $\chi=e, h, m_{\chi}^{*}$ is the (electron or hole) effective mass, and the plus (minus) sign refers to $\chi=e(\chi=h)$.

We numerically solve Eq. (1) for arbitrary confinement potential and field orientation by discretizing it on a homogeneous real-space grid of $N=N_{1} \times N_{2} \times N_{3}$ points, identified by the vectors $\mathbf{r}_{i}=\sum_{k=1}^{3}\left(\lambda_{i}^{k}-N_{k} / 2\right) \Delta_{k} \hat{\mathbf{e}}_{k}$, with $\lambda_{i}^{k}=1, \ldots, N_{k}$ and $\hat{\mathbf{e}}_{1,2,3}=\hat{\mathbf{x}}, \hat{\mathbf{y}}, \hat{\mathbf{z}}$. The resulting finite-difference equation can be rephrased in terms of the following discrete eigenvalue problem:

$$
\begin{gathered}
\sum_{j=1}^{N}\left\{\frac { 1 } { 2 m _ { \chi } ^ { * } } \left[-\hbar^{2}\left(\nabla^{2}\right)_{i j} \mp \frac{2 i \hbar|e|}{c}(\mathbf{A} \cdot \nabla)_{i j}\right.\right. \\
\left.\left.+\frac{e^{2}}{c^{2}}\left(\mathbf{A}^{2}\right)_{i j}\right]+V_{i j}^{\chi}\right\} \phi_{\alpha, j}^{\chi}=\epsilon_{\alpha}^{\chi} \phi_{\alpha, i}^{\chi},
\end{gathered}
$$

where $\phi_{\alpha, i}^{\chi}=\phi_{\alpha}^{\chi}\left(\mathbf{r}_{i}\right)$. The vector- and the confining-potential operators are diagonal in the real-space basis: $V_{i j}=\delta_{i j} V\left(\mathbf{r}_{i}\right)$ and $\left(\mathbf{A}^{2}\right)_{i j}=\delta_{i j}\left[\mathbf{A}\left(\mathbf{r}_{i}\right)\right]^{2}$, whereas the two differential operators act on the wave function vectors asfollows:

$$
\sum_{j=1}^{N}\left(\nabla^{2}\right)_{i j} \phi_{\alpha, j}=\sum_{k=1}^{3} \frac{\phi_{\alpha}\left(\mathbf{r}_{i}+\Delta_{k} \mathbf{e}_{k}\right)-2 \phi_{\alpha}\left(\mathbf{r}_{i}\right)+\phi_{\alpha}\left(\mathbf{r}_{i}-\Delta_{k} \mathbf{e}_{k}\right)}{\Delta_{k}^{2}},
$$

$$
\sum_{j=1}^{N}(\mathbf{A} \cdot \nabla)_{i j} \phi_{\alpha, j}=\sum_{k=1}^{3} A_{k, i} \frac{\phi_{\alpha}\left(\mathbf{r}_{i}+\Delta_{k} \mathbf{e}_{k}\right)-\phi_{\alpha}\left(\mathbf{r}_{i}-\Delta_{k} \mathbf{e}_{k}\right)}{2 \Delta_{k}},
$$

with $A_{k, i}=A_{k}\left(\mathbf{r}_{i}\right)$. The results discussed hereafter are obtained with $N_{1}=N_{2}=N_{3}=64$; the $262144 \times 262144$ sparse Hamiltonian matrix is diagonalized by means of the Lanczos algorithm.

The coupling of the magnetic field with the spin degrees of freedom are accounted for by the following Zeeman terms: ${ }^{13}$

$$
h_{\mathrm{spin}}^{\chi}\left(\mathbf{S}_{e}, \mathbf{J}_{h}\right)=\frac{\mu_{B}}{\hbar} \sum_{i=x, y, z} B_{i}\left(g_{e, i}^{*} S_{e, i}-\frac{g_{h, i}^{*}}{3} J_{h, i}\right),
$$

where the $g_{e, i}^{*}$ and $g_{h, i}^{*}$ are the elelctron and heavy-hole effective $g$ factors, respectively. However, the spin degrees of freedom mainly affect the few-particle states of our present concern through the statistics, rather than through the Zeeman splittings, which are suppressed by the optically induced antiparallel orientation of the electron and hole spins. ${ }^{14}$ Therefore, in the following the contribution of $h_{\text {spin }}^{\chi}\left(\mathbf{S}_{e}, \mathbf{J}_{h}\right)$ to the single- and few-particle energies will be neglected.

\section{B. Few-particle states}

In a second step, we include the Coulomb interactions between carriers. The second-quantization, full many-body Hamiltonian $\mathcal{H}$ is given by the sum of a single-particle part,

$$
\mathcal{H}_{s p}=\sum_{\chi=e, h} \sum_{\alpha=1}^{n_{\chi}} \epsilon_{\alpha}^{\chi} \hat{c}_{\chi, \alpha}^{\dagger} \hat{c}_{\chi, \alpha}
$$

and of the one accounting for the carrier-carrier Coulomb interactions, namely

$$
\begin{aligned}
\mathcal{H}_{c}= & \frac{1}{2} \sum_{\chi=e, h} \sum_{\alpha \beta \gamma \delta=1}^{n_{\chi}} V_{\alpha \beta \gamma \delta}^{\chi \chi} \hat{c}_{\chi, \alpha}^{\dagger} \hat{c}_{\chi, \gamma}^{\dagger} \hat{\gamma}_{\chi, \delta} \hat{c}_{\chi, \beta} \\
& +\sum_{\alpha \beta=1}^{n_{e}} \sum_{\gamma \delta=1}^{n_{h}} V_{\alpha \beta \gamma \delta}^{e h} \hat{c}_{\chi, \alpha}^{\dagger} \hat{c}_{\chi^{\prime}, \gamma}^{\dagger} \hat{c}_{\chi^{\prime}, \delta} \hat{c}_{\chi, \beta},
\end{aligned}
$$

where $n_{\chi}$ are the number of SP states that are taken into account. The results discussed in the following are obtained with $n_{e}=n_{h}=20$. The Coulomb matrix elements read

$$
V_{\alpha \beta \gamma \delta}^{\chi \chi^{\prime}}=\iint \frac{\left[\phi_{\alpha}^{\chi}(\mathbf{r})\right]^{*}\left[\phi_{\gamma}^{\chi^{\prime}}\left(\mathbf{r}^{\prime}\right)\right]^{*} \phi_{\delta}^{\chi^{\prime}}\left(\mathbf{r}^{\prime}\right) \phi_{\beta}^{\chi}(\mathbf{r})}{\kappa_{r}\left|\mathbf{r}-\mathbf{r}^{\prime}\right|} d \mathbf{r} d \mathbf{r}^{\prime},
$$

being $\kappa_{r}$ is the static dielectric constant of the semiconductor medium. These matrix elements are calculated by numerically integrating the following expression: 

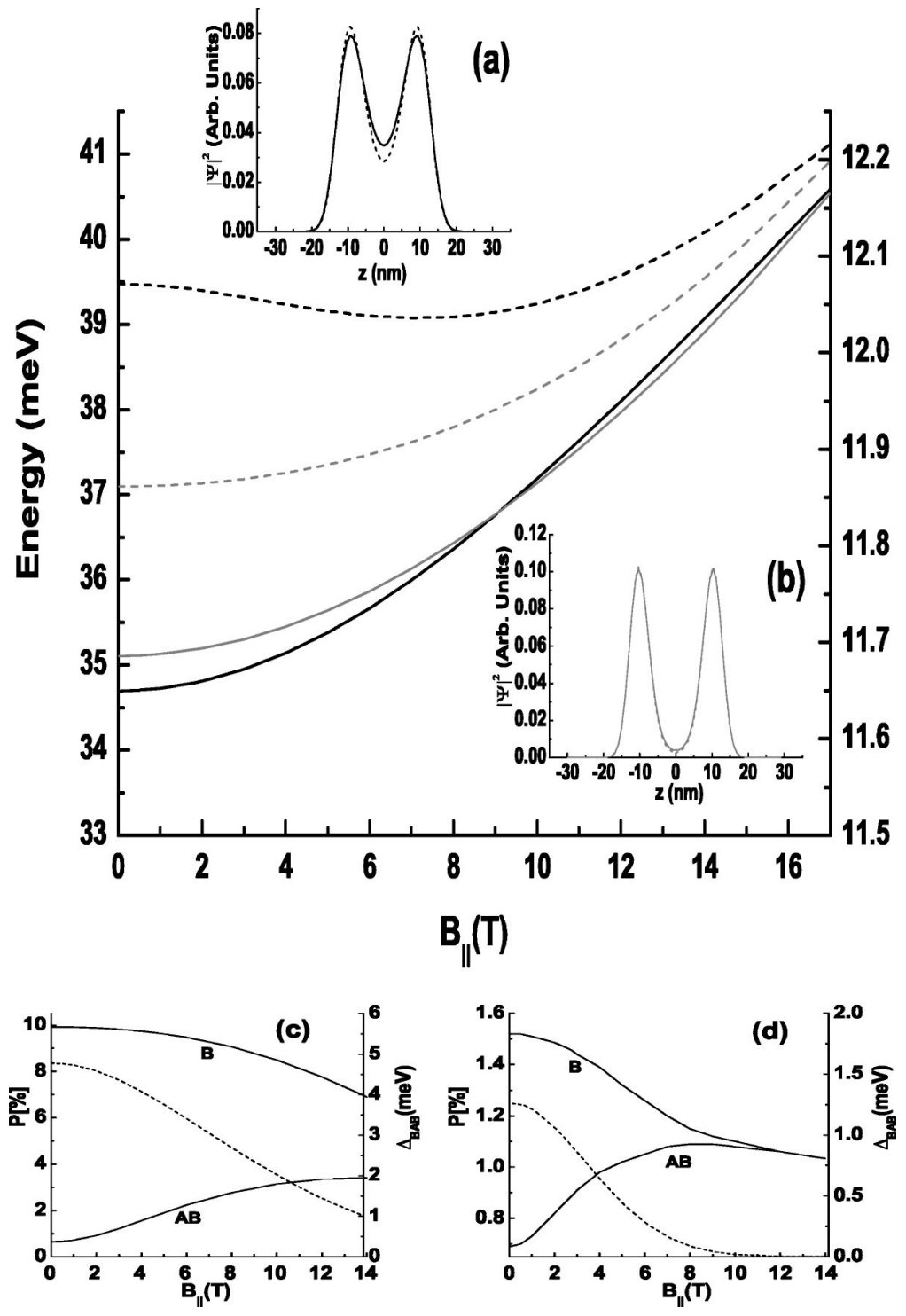

FIG. 1. Single-particle energies for electrons (left axis, black lines) and holes (right axis, gray lines) in a symmetric double QD, as a function of the in-plane magnetic field. The curves correspond to the $B$ (solid lines) and $A B$ (dashed lines) eigenstates. The insets show the square of the electron (a) and hole (b) $B$ wave functions along $z$ for two selected values of $B_{\|}$, namely $0 \mathrm{~T}$ (solid lines) and $15 \mathrm{~T}$ (dashed lines), for $x=y=0$. In the lower insets we plot the electron charge density integrated in the interdot barrier for the above confinement potential [panel (c)], as well as for a double-well (d) profile in the growth direction (solid lines, left axes), respectively; the magneticfield dependence of $\Delta_{B A B}$ is also shown (dotted lines, right axes). In the former case we identify the barrier with the region $|z|<a / \sqrt{3}$; in the latter case, the width of each well is $l=12 \mathrm{~nm}$, the width and height of the interdot barrier are $d=4 \mathrm{~nm}$ and $V_{0}^{e}=400 \mathrm{meV}$, respectively.

$$
V_{\alpha \beta \gamma \delta}^{\chi \chi^{\prime}}= \pm \frac{e^{2}}{\kappa} \int \mathcal{F}^{-1}\left[\frac{1}{k^{2}} \widetilde{\Phi}_{\alpha \beta}^{\chi}(\mathbf{k})\right] \Phi_{\gamma \delta}^{\chi^{\prime}}(\mathbf{r}) d \mathbf{r}
$$

where $\Phi_{\alpha \beta}^{\chi}(\mathbf{r})=\left[\phi_{\alpha}^{\chi}(\mathbf{r})\right]^{*} \phi_{\beta}^{\chi}(\mathbf{r})$, and $\widetilde{\Phi}_{\alpha \beta}^{\chi}(\mathbf{k})=\mathcal{F}\left[\Phi_{\alpha \beta}^{\chi}(\mathbf{r})\right]$ is its Fourier transform.

The overall, sparse Hamiltonian $\mathcal{H}=\mathcal{H}_{s p}+\mathcal{H}_{c}$ is directly diagonalized within a truncated Hilbert space generated by the $N_{\text {conf }}$ configurations of lowest SP energy $\left\langle\mathcal{H}_{s p}\right\rangle$ corresponding to fixed numbers of electrons $\left(N_{e}\right)$ and holes $\left(N_{h}\right)$, with defined spin orientations.

\section{Spatial correlation and optical properties}

In order to investigate the spatial correlations between any two carriers in the few-particle eigenstate $|\Psi\rangle$, we compute their spin-resolved pair correlation functions

$$
\begin{aligned}
g_{\chi \chi^{\prime}}^{\Psi}\left(\mathbf{r}_{1}, \mathbf{r}_{2}\right) & =\left\langle\Psi\left|\hat{\psi}_{\chi}^{\dagger}\left(\mathbf{r}_{1}\right) \hat{\psi}_{\chi^{\prime}}^{\dagger}\left(\mathbf{r}_{2}\right) \hat{\psi}_{\chi^{\prime}}\left(\mathbf{r}_{2}\right) \hat{\psi}_{\chi}\left(\mathbf{r}_{1}\right)\right| \Psi\right\rangle \\
& =\sum_{\alpha \beta=1}^{n_{\chi}} \sum_{\gamma \delta=1}^{n_{\chi^{\prime}}} \Phi_{\alpha \beta}\left(\mathbf{r}_{1}\right) \Phi_{\gamma \delta}\left(\mathbf{r}_{2}\right)\left\langle\Psi\left|\hat{c}_{\alpha}^{\dagger} \hat{c}_{\gamma}^{\dagger} \hat{c}_{\delta} \hat{c}_{\beta}\right| \Psi\right\rangle
\end{aligned}
$$

which gives the joint probability of finding carrier $\chi$ in $\mathbf{r}_{1}$ and carrier $\chi^{\prime}$ in $\mathbf{r}_{2}$, being $\left(\chi, \chi^{\prime}\right)=(e, h) \otimes(\uparrow, \downarrow)$ and $\hat{\psi}_{\chi}(\mathbf{r})$ the (electron or hole) field operator. As a further step, we integrate $g_{\chi \chi^{\prime}}\left(\mathbf{r}_{1}, \mathbf{r}_{2}\right)$ over the center-of-mass coordinates $\mathbf{R}=\frac{1}{2}\left(\mathbf{r}_{1}+\mathbf{r}_{2}\right)$,

$$
\bar{g}_{\chi \chi^{\prime}}^{\Psi}(\mathbf{r})=\int g_{\chi \chi^{\prime}}(\mathbf{R}+\mathbf{r} / 2, \mathbf{R}-\mathbf{r} / 2) d \mathbf{R}
$$

gives the probability of finding the two particles at a relative position $\mathbf{r}=\mathbf{r}_{1}-\mathbf{r}_{2}$.

As a final step, we compute the artificial molecule's linear optical properties, resulting from the interband transitions 
between different electron-hole complexes. The amplitude of such radiative transitions is given by the following expression:

$$
\mathcal{A}_{i \rightarrow f}=\left\langle\Psi_{f}\left|\sum_{\alpha=1}^{n_{e}} \sum_{\beta=1}^{n_{h}} M_{\alpha \beta} \hat{c}_{e, \alpha} \hat{c}_{h, \beta}\right| \Psi_{i}\right\rangle,
$$

where $M_{\alpha \beta} \propto \int \phi_{\alpha}^{e}(\mathbf{r}) \phi_{\beta}^{h}(\mathbf{r}) d \mathbf{r}$, while $\left|\Psi_{i}\right\rangle$ and $\left|\Psi_{f}\right\rangle$ are the initial and final states, respectively. The luminescence spectra are finally obtained by summing over different initial $\left|\Psi_{\lambda}^{N_{e}, N_{h}}\right\rangle$ and final states $\left|\Psi_{\lambda^{\prime}}^{N_{e}-1, N_{h}-1}\right\rangle$, the former being thermally distributed according to the Boltzmann distribution,

$$
I(\omega, T)=\sum_{\lambda, \lambda^{\prime}}\left|\mathcal{A}_{\lambda \rightarrow \lambda^{\prime}}\right|^{2} \mathcal{D}\left(\hbar \omega-E_{\lambda}+E_{\lambda^{\prime}}\right) e^{-E_{\lambda} / k_{B} T},
$$

with the Lorentzian broadening of the emission line $\mathcal{D}\left(\hbar \omega-E_{\lambda}+E_{\lambda^{\prime}}\right)=2 \gamma /\left(\omega^{2}+\gamma^{2}\right)$ determined by the phenomenological paramenter $\gamma$.

\section{RESULTS}

In this section we summarize our understanding of the artificial molecule's field-dependent properties. We start by discussing the SP properties (Sec. III A): These provide the ingredients for the calculation and interpretation of the interacting states, which include neutral and charged excitons (Sec. III B), as well as biexcitons (Sec. III C). We finally show the optical response (luminescence spectra) of the system which results from the radiative recombination of the above electron-hole complexes (Sec. III D).

\section{A. Single particle states}

Within our prototypical model of an artificial molecule, the spatial confinement of the carriers is accounted for by a separable confining potential $V(\rho, z)=V_{p l}(\rho)+V_{v}(z)$, where $\rho^{2}=x^{2}+y^{2}$. The in-plane and the vertical terms read: ${ }^{15}$

$$
\begin{gathered}
V_{p l}(\rho)=\frac{1}{2} m * \omega_{x y}^{2} \rho^{2}, \\
V_{v}(z)=\frac{m^{*} \omega_{z}^{2}}{8 a^{2}}\left(z^{2}-a^{2}\right)^{2} .
\end{gathered}
$$

The in-plane components of the wave functions, resulting from the 2D parabolic confinement, are the Fock-Darwin states, which are known to produce results in good agreement with the experiments, especially as far as the lowest shells are concerned. ${ }^{1}$ In the absence of an in-plane magnetic field, the interdot coupling only depends on $V_{v}$ : In particular, $a$ determines both the distance between the centers of the two dots, $d=2 a$, and the maximum height of the interdot barrier, $V_{v}(0)=m * \omega_{z}^{2} a^{2} / 8$. Hereafter, we consider two GaAs/AlGaAs coupled QDs desciribed by the following material and confinement parameters: $m_{e}^{*}=0.067 m_{0}$, $m_{h}^{*}=0.38 m_{0}, \quad \kappa_{r}=12.9, \quad \hbar \omega_{0}^{e}=15 \mathrm{meV}, \quad \hbar \omega_{0}^{h}=4.6 \mathrm{meV}$, $\hbar \omega_{z}^{e}=50 \mathrm{meV}, \hbar \omega_{z}^{h}=15.4 \mathrm{meV}, a=5.5 \mathrm{~nm}$. As discussed in greater detail below, these structural parameters provide a coherent interdot coupling for both electrons and holes; be- sides, the lowest excitations associated to the motion in the growth direction are comparable to the energy scales characterizing a magnetic field of achieveable intensity, as required for the discussed behaviors to be experimentally observable.

The in-plane magnetic field $\mathbf{B}=B_{\|} \hat{\mathbf{x}}$ couples the degrees of freedom corresponding to the $x y$ and to the $z$ directions, and reduces the symmetries of the system: In fact, while $h_{\text {orb }}\left(B_{\|}=0\right)$ commutes with the reflection operators $A_{x}, A_{y}$ and $A_{z}, h_{\text {orb }}\left(B_{\|} \neq 0\right)$ is only symmetric with respect to the reflection about the $y z$ plane and the $x$ axis. Figure 1 shows the energies of the bonding $(B)$ and antibonding $(A B)$ orbitals, namely the ground- and first excited-state, as a function of $B_{\|}$. At zero field, both states are characterized by an azimuthal quantum number $m=0$ ( $s$ shell), and are either symmetric $\left(A_{z}=+1\right)$ or antisymmetric $\left(A_{z}=-1\right)$ with respect to the reflection about the $x y$ plane: Therefore, $|0\rangle=\left|m=0, A_{z}=+1\right\rangle$ and $|1\rangle=|0,-1\rangle$. The field is seen to suppress the energy splitting $\Delta_{B A B} \equiv \epsilon_{A B}-\epsilon_{B}=\epsilon_{1}-\epsilon_{0}$ for both electrons and holes (black and gray lines, respectively), while no sensible change is observed in the profile of the $B$ and $A B$ wave functions along $x=y=0$ [Figs. 1(a) and 1(b)]. The clear asymmetry between electrons and holes is due to the larger effective mass of the latter, which divides all the kinetic terms in $h_{\text {orb }}(\mathbf{r})$, thus resulting in a larger localization of the holes within each dot (insets of Fig. 1) and in a smaller effect of the magnetic field. In order to clarify the relation between $\Delta_{B A B}$ and the carrier distribution at the interdot barrier, we have integrated the electron charge density in this region and plotted the resulting percentage $P$ as a function of $B_{\|}$. The field increase from 0 to $14 \mathrm{~T}$ reduces the difference between $P_{B}$ and $P_{A B}$ from $10 \%$ to $2 \%$ [Fig. 1(c)]. However, unlike the case where $\Delta_{B A B}$ is suppressed by increasing the barrier width, here the two dots are quantum-mechanically coupled even in the limit of a vanishing energy splitting. Figure 1(d) corresponds to a double-well profile of the confinement potential in the growth direction: in spite of the quantitative differences between the two cases, which depend on the different barrier smoothnesses and $\Delta_{B A B}$ values at zero field, the convergence of $P_{B}$ and $P_{A B}$ to a finite value clearly turns out to be a general feature.

A deeper insight into the magnetic-field effect can be provided by the average values of the field-dependent terms in the SP Hamiltonian (Fig. 2),

$$
\delta\left(B_{\|}\right) \equiv\left\langle h_{\mathrm{orb}}-h_{\mathrm{orb}}\left(B_{\|}=0\right)\right\rangle=\frac{e^{2} B_{\|}^{2}}{8 m * c^{2}}\left\langle z^{2}+y^{2}\right\rangle \pm \frac{e B_{\|}}{2 m * c}\left\langle L_{x}\right\rangle,
$$

where $L_{x}=y p_{z}-z p_{y}$. The first term (denoted by circles in the figure) gives rise to a diamagnetic contribution, which tends to increase $\Delta_{B A B}$. In fact, the average value of $\left\langle y^{2}+z^{2}\right\rangle$ is systematically higher for the $A B$ state (dotted line) than for the $B$ one (solid line). On the contrary, the second term (squares) energetically favors the $A B$ orbital and dominates over the first one, thus contributing to the suppression of the splitting (see the triangles in Fig. 2). In first-order perturbation theory no paramagnetic effect is induced by the field on any $L_{x}=0$ state, such as the $\phi_{0,1}^{e}(\mathbf{r})$ that we 


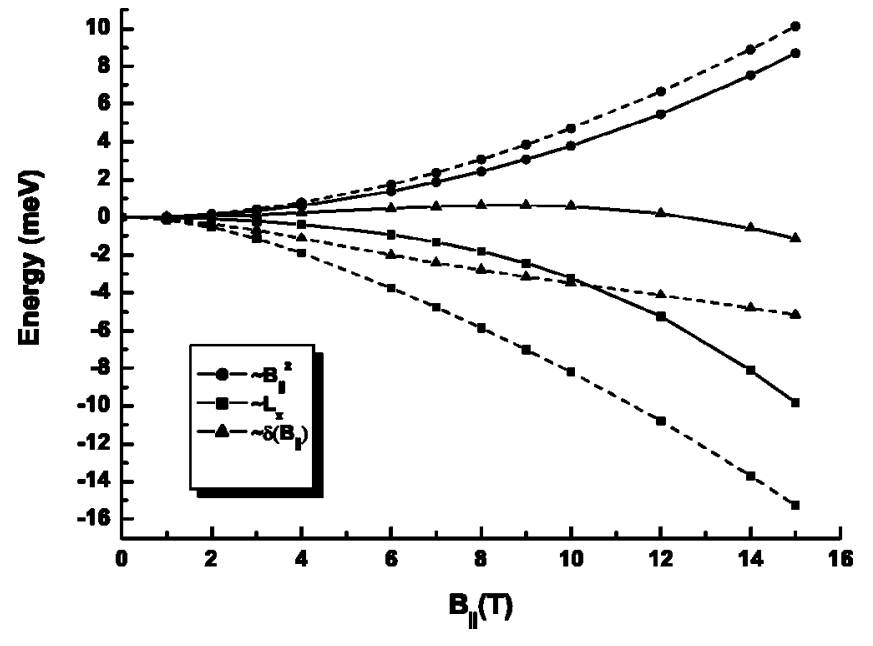

FIG. 2. Expectation values of the field-dependent terms in the single-electron Hamiltonian of Eq. (1): The solid (dashed) lines correspond to the $B(A B)$ states; circles, squares, and triangles stand for the diamagnetic part, the paramagnetic one, and the overall expectation value, respectively.

are presently considering. However, the mixing with higher states implies, already at the second-order level, a paramagnetic contribution (van Vleck paramagnetism ${ }^{16}$ ) $\Delta E=-\left(e B_{\|} / 2 m * c\right)^{2} \Sigma_{n}\left|\left\langle 0\left|L_{x}\right| n\right\rangle\right|^{2} /\left(\epsilon_{n}-\epsilon_{0}\right)$. This expression provides an intuitive understanding of the above behaviors. In fact, due to the system symmetries and to the energy splittings at the denominator, the main contributions to $\Delta E$ of the $|0\rangle=\left|m=0, A_{z}=+1\right\rangle$ and $|1\rangle=|0,-1\rangle$ states are negative and, respectively, proportional to $\left|\left\langle 0,+1\left|L_{x}\right| \pm 1,-1\right\rangle\right|^{2} /\left(\hbar \omega_{x y}\right.$ $\left.+\Delta_{B A B}\right)$ and $\left|\left\langle 0,-1\left|L_{x}\right| \pm 1,+1\right\rangle\right|^{2} /\left(\hbar \omega_{x y}-\Delta_{B A B}\right)$, the latter being systematically larger than the former (the two matrix elements at the numerator are identical). The above expressions also suggest that a strong in-plane confinement (i.e., a large $\left.\hbar \omega_{x y}\right)$ hinders the field-induced suppression of $\Delta_{B A B}$. The effect of the field on the hole states (not shown) is reduced in magnitude, but qualitatively the same as the one discussed for the electrons.

In order to verify possible effects of the barrier finiteness, as well as the robustness of our findings with respect to the molecule's composition profile, we have performed further calculations for Gaussian QDs: ${ }^{17}$

$$
V^{\chi}(\mathbf{r})=V_{0}^{\chi}\left\{1-\sum_{i=1}^{2} \exp \left[-\frac{\left(x^{2}+y^{2}\right)}{2 l_{x y}^{2}}-\frac{\left(z-z_{0, i}\right)^{2}}{2 l_{z}^{2}}\right]\right\},
$$

where $V_{0}^{\chi=e, h}$ are the electron and hole band-offsets, respectively. The results, shown in Ref. 19, show no qualitative differences with respect to the ones discussed hereafter. This is also due to the fact that intradot excitations in the growth direction are not involved in the few-particle states of our present concern (the $B$ and $A B$ states arise from the hybridization of the single-dot ground-state wave functions along $z$ ): the carriers are, therefore, rather unsensitive to the detailed shape of $V(z)$ away from the local minima.

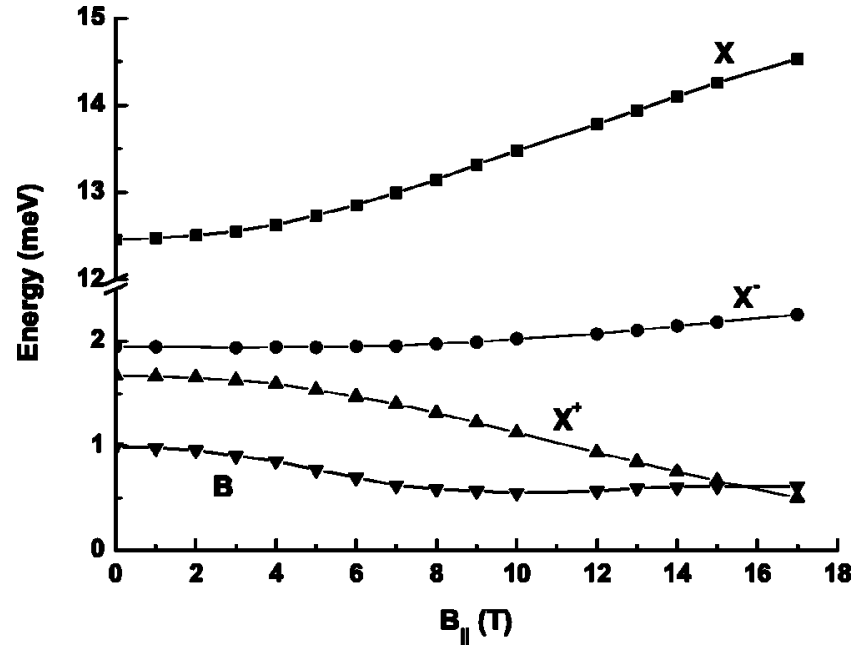

FIG. 3. The binding energies of the neutral $(X)$ and charged $\left(X^{-}\right.$ and $X^{+}$) excitons, and of the biexciton $B$ as a function of the inplane magnetic field $B_{\|}$are denoted by squares, circles, upward- and downward-oriented triangles, respectively.

\section{B. Neutral and charged excitons}

Due to the 3D confinement of the carriers, the fewparticle states in single- and coupled-QDs are strongly affected by the Coulomb interactions, which typically induce large energy renormalizations, as compared to heterostructures of higher dimensionality. We start by considering the exciton binding energy, which is defined as $\Delta_{X} \equiv \epsilon^{e}+\epsilon^{h}-E_{X}$, where $\epsilon_{0}^{e}, \epsilon_{0}^{h}$, and $E_{X}$ are the electron, hole, and exciton ground-state energies, respectively. As shown in Fig. 3 (squares), the exciton binding energy monothonically increases as a function of the in-plane magnetic field $B_{\|}$. This behavior is due to the combination of different effects: The wave function squeezing, resulting in an enhanced modulus of the attractive Coulomb matrix element, $\left|V_{1111}^{\text {eh }}\right|$; an increase of $\epsilon_{0}^{e}+\epsilon_{0}^{h}$ which is larger than that of $E_{s p}=\left\langle X\left|\mathcal{H}_{s p}\right| X\right\rangle$, due to the presence of a decreasing $A B$ component $\left|\phi_{1}^{e}\right\rangle \otimes\left|\phi_{1}^{h}\right\rangle$ in the latter; the suppression of the energy splitting $\Delta_{B A B}$, which favors the interdot correlation and thus further enhances the Coulomb interaction between the carriers, as explained in greater detail below. Figure 4(b) shows the $z$ dependence of the electron-hole pair-correlation function for the exciton ground state $|X\rangle, \bar{g}_{e h}^{X}(x=0, y=0, z): \bar{g}_{e h}^{X}$ is strongly peaked around $z=0$ already at $B_{\|}=0$ (solid line), and this feature is further enhanced at high magnetic field ( $B_{\|}=15 \mathrm{~T}$, dotted line); correspondingly, the interdot correlation between the electron and the hole suppresses the probability of finding the two carriers in different dots. The spatial correlation in the $z$ direction reduces the Coulomb energy $E_{c}=\left\langle X\left|\mathcal{H}_{c}\right| X\right\rangle$ at the cost of occupying the $A B$ orbitals: Therefore, the correlated exciton state $|X\rangle \simeq\left|\phi_{0}^{e}\right\rangle \otimes\left|\phi_{0}^{h}\right\rangle+\left|\phi_{1}^{e}\right\rangle$ $\otimes\left|\phi_{1}^{h}\right\rangle$ is favored by the magnetic-field induced suppression of $\Delta_{B A B}$.

The charging of the exciton, be it negative $\left(X^{-}\right)$or positive $\left(X^{+}\right)$, strongly affects its magnetic-field dependence, for it introduces a competition between the attractive and the repulsive Coulomb interactions, thus widening the variety of 


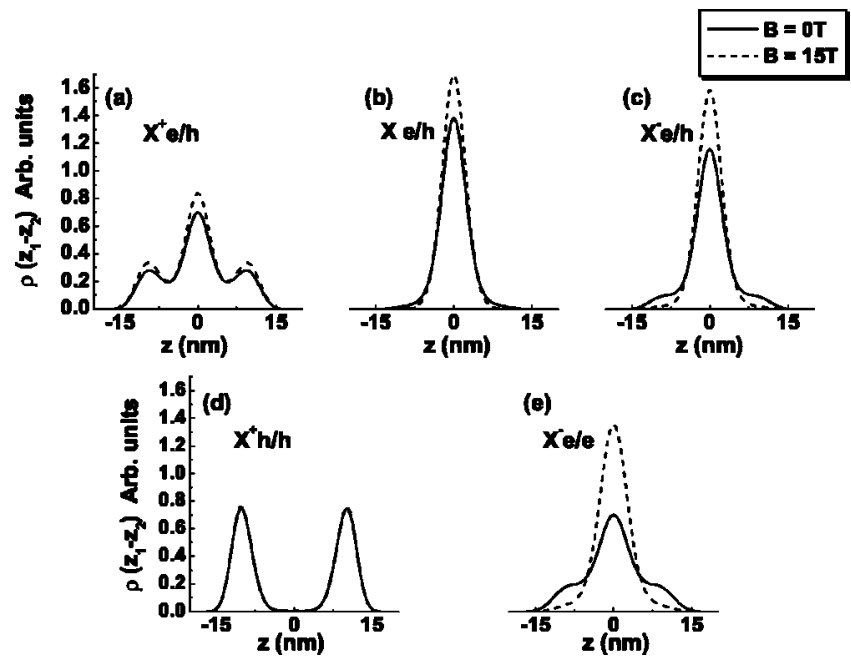

FIG. 4. Projection along $z$ of the pair-correlation functions, $\bar{g}_{\chi \chi^{\prime}}(0,0, z)$, of the neutral and charged excitons, for two selected values of the in-plane magnetic field, namely $B_{\|}=0 \mathrm{~T}$ and $B_{\|}=15 \mathrm{~T}$ (solid and dotted lines, respectively). In detail, In panels (a)-(c) $\chi=e$ and $\chi^{\prime}=h$, whereas $\chi=\chi^{\prime}=h$ in (d) and $\chi=\chi^{\prime}=e$ in (e); panels (a) and (d) refer to $X^{+},(\mathrm{c})$ and (e) to $X^{-}$and (b) to $X$.

the carrier localizations and correlations that can in principle minimize the overall energy. In analogy to what has been done for the neutral exciton, we define for $X^{+(-)}$the binding energy

$$
\Delta_{X^{+(-)}} \equiv \epsilon_{0}^{h(e)}+E_{X}-E_{X^{+(-)}},
$$

where $E_{X^{+(-)}}$is the energy of the positive (negative)-trion ground state. We prefer the above definition to, e.g., $\Delta_{X^{+(-)}}^{\prime} \equiv 2 \epsilon_{0}^{h(e)}+\epsilon_{0}^{e(h)}-E_{X^{+(-)}}$, for it provides a more intuitive understanding of the effects produced on the exciton by the addition of an extra hole (electron); besides, unlike $\Delta_{X^{ \pm}}^{\prime}, \Delta_{X^{ \pm}}$ is directly accessible from an experimental point of view, for it corresponds to the difference between the recombination energies of the neutral and of the charged excitons, $\hbar \omega_{X}-\hbar \omega_{X^{+(-)}}=E_{X}-\left[E_{X^{+(-)}}-\epsilon_{0}^{h(e)}\right]$. As shown in Fig. 3 (circles), $\Delta_{X^{-}}$is always positive, and weakly increases with increasing $B_{\|}$. The interpretation for this behavior is provided by the electron-hole and electron-electron pair-correlation functions plotted in Figs. 4(c) and 4(e), respectively. The increased interdot correlation resulting from the application of the magnetic field (dotted lines) favors the carrier localization, whereas the balance between the attractive and repulsive interactions induces the carriers to localize in a same dot: This is shown by the disappearence of the side peaks in both $\bar{g}_{e h}^{X^{-}}$and $\bar{g}_{e e}^{X^{-}}$. The exciton and the additional electron, therefore, form a bound system.

The binding energy $\Delta_{X^{+}}$of the positively charged exciton, instead, decreases with increasing $B_{\|}$(see Fig. 3). Correspondingly, the carriers tend to minimize the Coulomb repulsion by avoiding a double occupation of each dot [Fig. 4(d)], rather than maximizing the attractive interaction; the electron is delocalized over the whole molecule and symmetrically distributed between the two dots [Fig. 4(a)]. Such striking difference between $X^{-}$and $X^{+}$can be traced back to the fact that hole wave functions are more strongly confined than the electron ones: ${ }^{18}$ The extra hole is, therefore, more strongly repealed by the other hole than it is attracted by the electron, and this prevents it from forming a bound state with the exciton. The comparison between the solid and the dotted lines in Figs. 4(a) and 4(d) actually shows that the field does not induce meaningful changes in the properties of $X^{+}$: In fact, the energetic cost of the hole-hole correlation, namely $\Delta_{B A B}^{h}$, is already small enough for $B_{\|}=0$; besides, $\Delta_{B A B}^{e}$ does not play any meaningful role in the positively chargedexciton behavior, being the electron-hole correlation negligible even in the high-field regime [Fig. 4(a)]. Being the positively charged exciton relatively insensitive to the field, the decreasing trend of $\Delta_{X^{+}}$as a function of $B_{\|}$can be essentially traced back to the large diamagnetic shift affecting the "reference" exciton energy $E_{X}$.

Such qualitative differences in the magnetic-field dependence of the binding energies $\Delta_{X}, \Delta_{X^{+}}$, and $\Delta_{X^{-}}$recall the ones that occur in their dependence on the interdot distance, ${ }^{10}$ thus suggesting the analogous role played in this respect by the physical quantities $B_{\|}$and $d$. In particular, the results from the interplay between the interdot tunneling, the in-plane field, and intradot Coulomb interactions and, therefore, provide a clear fingerprint of both the coherent interdot coupling and of the exciton charging.

\section{Biexcitons}

In analogy to what has been done for the charged excitons, we define the biexciton binding energy as

$$
\Delta_{B} \equiv 2 E_{X}-E_{B},
$$

which quantifies the contribution of the exciton-exciton interaction to $E_{B}$. In fact, in the absence of such Coulomb interaction, the multiexciton states would follow an aufbau logic, and progressively occupy exciton states of incresing energy: This justifies the use of $2 E_{X}$ as a reference for the biexciton energy. The correctness of the noninteracting picture can be quantified by $\left|\left\langle B\left|\hat{I}_{X^{\dagger}}^{\dagger} \hat{T}_{X^{\dagger}}^{\dagger}\right| 0\right\rangle\right|$, where the creation of an optically active exciton $|X\rangle=\sum_{\alpha=1}^{n_{e}} \sum_{\beta=1}^{n_{h}} c_{\alpha \beta}^{X}\left|\phi_{\alpha}^{e}\right\rangle \otimes\left|\phi_{\beta}^{h}\right\rangle$ (total angular momentum $M= \pm 1$ ) is obtained by applying to the vacuum state $\hat{I}_{X^{\uparrow(\downarrow)}}^{\dagger}=\Sigma_{\alpha, \beta} c_{\alpha \beta}^{X} \hat{c}_{e, \alpha \downarrow(\uparrow)}^{\dagger} \hat{c}_{h, \beta \uparrow(\downarrow)}^{\dagger}$, and $\hat{c}_{e, \alpha \uparrow(\downarrow)}^{\dagger}$ $\left(\hat{c}_{h, \alpha \uparrow(\downarrow)}^{\dagger}\right)$ creates an electron (hole) with $s_{z}= \pm 1 / 2\left(j_{z}\right.$ $= \pm 3 / 2$ ). As discussed in more detail in the following, this picture fails in the case when the higher-order correlations set in.

The dependence of the biexciton-binding energy on $B_{\|}$is plotted in Fig. 3 (downward-oriented triangles). Unlike the neutral- and charged-excitons binding energies, $\Delta_{B}$ does not monotonically depend on the field: In fact, a minimum is seen to occur at $B_{\|} \sim 8 \mathrm{~T}$. The two trends on the low- and high-field sides of the minimum correspond to different kinds of carrier correlations characterizing the biexciton ground state, as shown in Fig. 5. At $B_{\|}=0$ (solid lines) the interdot correlation mainly occurs between identical carriers and minimizes the repulsive contributions to the overall Coulomb energy $\left\langle B\left|\mathcal{H}_{c}\right| B\right\rangle$. The double-occupation probability is suppressed for holes [Fig. 5(a)] and reduced for electrons 


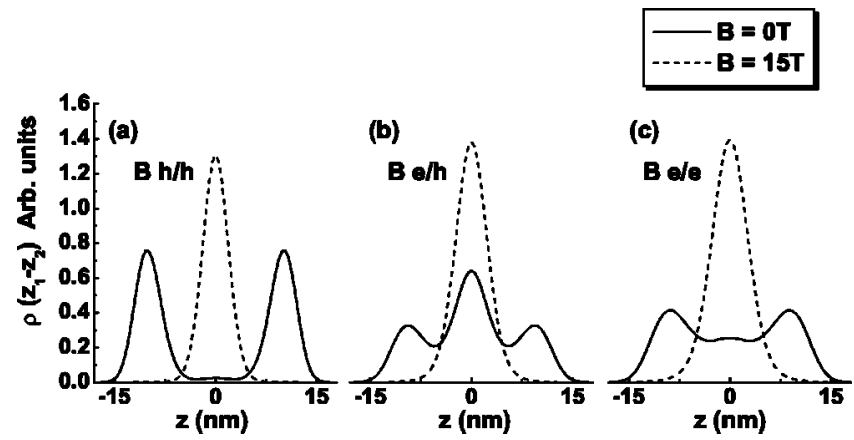

FIG. 5. Projection along $z$ of the hole-hole [panel (a)], electronhole (b), and electron-electron (c) pair-correlation functions for the biexciton ground state $|B\rangle$, for $B_{\|}=0 \mathrm{~T}$ and $B_{\|}=15 \mathrm{~T}$ (solid and dotted lines, respectively).

[Fig. 5(c)], the asymmetry between the two carriers arising from the different values of the energy splitting $\Delta_{B A B}^{e}$ and $\Delta_{B A B}^{h}$; electrons and holes, instead, are essentially uncorrelated [Fig. 5(b)] with each other. Correspondingly, the dominant configurations in the biexciton state vector $|B\rangle$ are $\left|\phi_{0}^{e}, \phi_{0}^{e}\right\rangle \otimes\left|\phi_{0}^{h}, \phi_{0}^{h}\right\rangle$ and $\left|\phi_{1}^{e}, \phi_{1}^{e}\right\rangle \otimes\left|\phi_{1}^{h}, \phi_{1}^{h}\right\rangle$. The application of the in-plane field drastically changes the correlation properties of $|B\rangle$. As shown by the dotted lines in Figs. 5(a)-5(c), for $B_{\|}=15 \mathrm{~T}$ all the pair-correlation functions become strongly peaked around $z=0$, i.e., all the carriers tend to localize in the same dot and the two excitons do form a bound state.

A closer inspection at the first excited biexciton states $\left|B_{1-3}\right\rangle$ shows how the above transition corresponds to the occurence of anticrossings (crossings) between quantum states with identical (different) symmetries. In Fig. 6 we plot the dependence on $B_{\|}$of $\left\langle B_{i}\left|\mathcal{H}_{s p}\right| B_{i}\right\rangle$ [Fig. 6(d)] and of the different Coulomb contributions $\left\langle B_{i}\left|V_{\chi \chi^{\prime}}\right| B_{i}\right\rangle$, with $i=0,1,2,3$ and $\chi \chi^{\prime}=e h, e e, h h$ [Figs. 6(a)-6(c), respectively; hereafter $\left|B_{0}\right\rangle$ denotes the biexciton ground state]. All four states are characterized by an even parity and $S_{e}=0$; all of them but $B_{1}$ also have $S_{h}=0$. In agreement with the Wigner-von Neumann no-crossing rule, the states $\left|B_{0}\right\rangle$ and $\left|B_{3}\right\rangle$, which share the same quantum numbers, anticross at
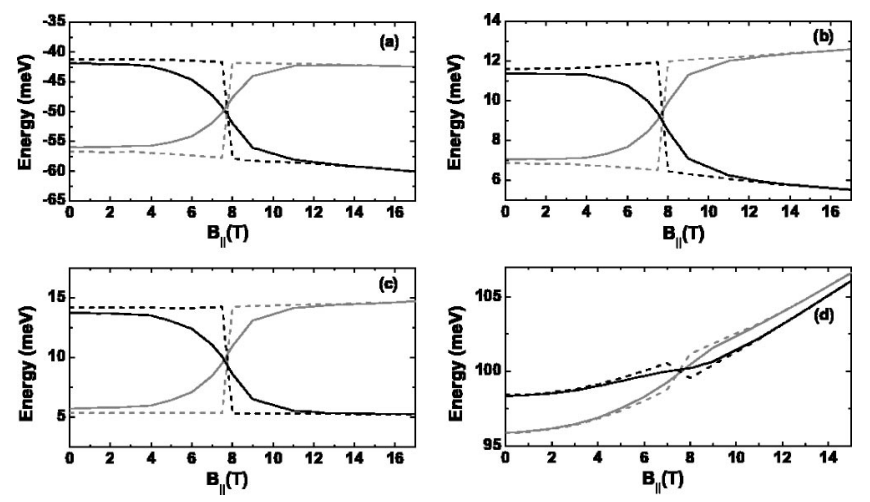

FIG. 6. Average values of the Coulomb terms $\left\langle B_{i}\left|V_{\chi \chi^{\prime}}\right| B_{i}\right\rangle$ [panels ((a)-(c) correspond to $\chi \chi^{\prime}=e h, e e, h h$ ] and of $\left\langle B_{i}\left|\mathcal{H}_{s p}\right| B_{i}\right\rangle$ [panel (d)] for the biexciton ground and first excited-states $\left|B_{i}\right\rangle$; the solid and dotted gray (black) lines correspond to the states $\left|B_{0}\right\rangle$ and $\left|B_{1}\right\rangle$ $\left(\left|B_{3}\right\rangle\right.$ and $\left.\left|B_{2}\right\rangle\right)$, respectively.

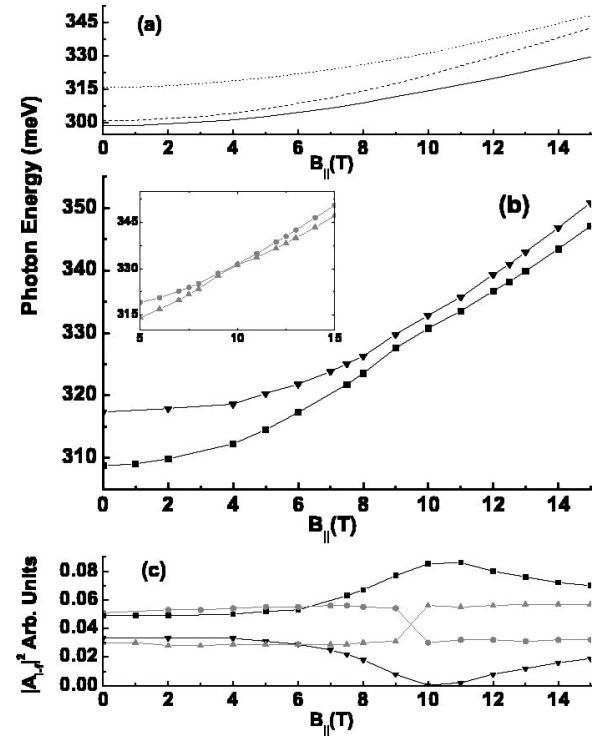

FIG. 7. Optical transitions as a function of the magnetic field $B_{\|}$. (a) Emission energies from the groundstates of the neutral and charged excitons $X, X^{+}$, and $X^{-}$(dotted, dashed, and solid lines, respectively). (b) Emission energies from the biexciton states $\left|B_{0}\right\rangle$ (squares) and $\left|B_{3}\right\rangle$ (downwards-oriented triangles): The two lines anticross at $B_{\|} \sim 8 \mathrm{~T}$. Inset, crossing between the lines corresponding to the biexciton states $\left|B_{1}\right\rangle$ (upwards-oriented triangles) and $\left|B_{2}\right\rangle$ (circles). (c) Squared moduli of the amplitudes $\mathcal{A}_{i \rightarrow f}$; the initial states correspond to $\left|B_{i}\right\rangle$, with $i=0,1,2,3$, and the same conventions as above for the symbols; the final states are the exciton ground- (decay from $\left|B_{0}\right\rangle$ and $\left|B_{3}\right\rangle$ ) and first excited-states $\left(\left|B_{1}\right\rangle\right.$ and $\left.\left|B_{2}\right\rangle\right)$.

$B_{\|} \sim 8 \mathrm{~T} ;\left|B_{1}\right\rangle$ and $\left|B_{2}\right\rangle$, instead, cross, for a very close value of $B_{\|}$. Correspondingly, the states $0-3$ and 1-2 swap the respective values of the different energetic contributions: In particular, the increases (decreases) of the $\left|\left\langle B_{i}\left|V_{\chi \chi^{\prime}}\right| B_{i}\right\rangle\right|$ occurs by localization of the corresponding carriers in the same (different) QD. This behavior qualitatively resembles the one discussed in Ref. 20, where the physical parameter that tunes the coupling regime is the width of the interdot barrier, and the profile of the confinement potential in the growth direction is given by a double quantum well. Similar results were also obtained for Gaussian QDs, ${ }^{19}$ thus demonstrating the robustness of above behaviors with respect to the details of the confinement potential.

\section{Optical properties}

As a final step, we consider the magnetic-field dependence of the artificial-molecule optical response. In particular, the luminescence spectra at low temperatures are dominated by the emission from the ground states of the different electron-hole complexes. In Fig. 7(a) we show the photon energies corresponding to similar transitions, namely $|X\rangle \rightarrow|0\rangle, \quad\left|X^{+}\right\rangle \rightarrow\left|\phi_{0}^{h}\right\rangle$ and $\left|X^{-}\right\rangle \rightarrow\left|\phi_{0}^{e}\right\rangle$, where $|0\rangle$ is the vacuum state. The diamagnetic shift, which characterizes all three transitions, is enhanced upon positive charging: Such increased energy of the recombining electron-hole pair, as compared to the other two cases, can be explained in terms 
of the already mentioned rigidity of $X^{+}$, whose interdot correlation properties are unaffected by $B_{\|}$.

The occurence of transitions between different correlation regimes as a function of the magnetic field shows up in the dependence on $B_{\|}$of the biexciton emission energy. In particular, the states $\left|B_{0}\right\rangle$ and $\left|B_{3}\right\rangle$ share the even parity, as well as the electron and hole total spins $\left(S_{e}=0, S_{h}=0\right)$; besides, the final state maximizing the oscillator strength $\mathcal{A}_{i \rightarrow f}$ is in both cases the exciton ground state $|X\rangle$. The anticrossing between the energy levels $E_{B_{0}}\left(B_{\|}\right)$and $E_{B_{3}}\left(B_{\|}\right)$(not shown here) is reflected by that involving the respective optical-transition energies, $E_{B_{0}}-E_{X}$ and $E_{B_{3}}-E_{X}$ [see Fig. 7(b)]. At the anticrossing, the $\left|B_{0}\right\rangle \rightarrow|X\rangle$ picks up the oscillator strength of $\left|B_{3}\right\rangle \rightarrow|X\rangle$, as shown in panel (c). In the same range of magnetic-field values, an analogous transition occurs between the two first excited biexciton states, $\left|B_{1}\right\rangle$ and $\left|B_{2}\right\rangle$, both characterized by an odd parity and by $S_{e}=0$, but with different hole spin $\left(S_{h}=1\right.$ and $S_{h}=0$, respectively, at $\left.B_{\|}=0\right)$. In Fig. 7(b) we show the photon energies corresponding to the $\left|B_{i=1,2}\right\rangle \rightarrow\left|X_{1}\right\rangle$, where $\left|X_{1}\right\rangle$, the exciton first excited state, maximizes $\mathcal{A}_{i \rightarrow f}$ for both biexcitons: due to the different symmetries of the corresponding eigenstates, the curves $E_{B_{1}}\left(B_{\|}\right)$and $E_{B_{2}}\left(B_{\|}\right)$are allowed to cross, and so do the displayed photon energies. The field dependence of the oscillator strength is strikingly different with respect to the previous case: In fact, as for the average values of the Coulomb and kinetic terms in the Hamiltonian, the two optical transitions simply swap the respective values of $\left|\mathcal{A}_{i \rightarrow f}\right|^{2}$.

\section{CONCLUSIONS}

To summarize, we have investigated the dependence on the in-plane magnetic field $B_{\|}$of the exciton and biexciton states in two vertically coupled QDs. The main effect of the field is that of reducing the energy splitting $\Delta_{B A B}$ between the $B$ and $A B$ states, without suppressing the coherent coupling of the dots, and thus enhancing the interdot correlations between the carriers. The neutral- $(X)$ and chargedexciton $\left(X^{+}, X^{-}\right)$binding energies monotonically depend on $B_{\|}$; however, the sign of such dependence, as well as the bound or unbound nature of the charged complex, critically depends on the charging. The biexciton, instead, undergoes a transition between different quantum states and correlation regimes as function of $B_{\|}$; the two excitons correspondingly pass from an unbound (low field) to a bound (high field) configuration. Such transition shows up in the optical response of the system, through the occurence of an anticrossing between the optical-transitions energies involving the biexciton ground state.

\section{ACKNOWLEDGMENTS}

We are grateful to Andrea Bertoni and Massimo Rontani for helpful discussion. This work has been partially supported by the projects MIUR-FIRB No. RBAU01ZEML, MIUR-COFIN 2003 No. 2003020984, INFM I.T. Calcolo Parallelo 2004, and MAE, Dir. Gen. per la Promozione e la Cooperazione Culturale.
*Electronic address: dbellucci@unimore.it

URL: www.nanoscience.unimore.it

${ }^{1}$ L. Jacak, P. Hawrylak, and A. Wois, Quantum Dots (Springer, Berlin, 2000).

${ }^{2}$ F. Troiani, U. Hohenester, and E. Molinari, Phys. Rev. B 62, R2263 (2000); E. Biolatti, I. D’ Amico, P. Zanardi, and F. Rossi, ibid. 65, 075306 (2002), and references therein.

${ }^{3}$ See, e.g., S. Watanabe, E. Pelucchi, B. Dwir, M. H. Baier, K. Leifer, and E. Kapon, Appl. Phys. Lett. 84, 2907 (2004).

${ }^{4}$ A. Zrenner, J. Chem. Phys. 112, 7790 (2000).

${ }^{5}$ M. Bayer, P. Hawrilak, K. Hinzer, S. Fafard, M. Korkusinski, Z.R. Wasilewski, O. Stern, and A. Forchel, Science 291, 451 (2001).

${ }^{6}$ V. Halonen, T. Chakraborty, and P. Pietiläinen, Phys. Rev. B 45, 5980 (1992); A. Wojs, P. Hawrylak, S. Fafard, and L. Jacak, ibid. 54, 5604 (1996); R. Rinaldi, P.V. Giugno, R. Cingolani, H. Lipsanen, M. Sopanen, J. Tulkki, and J. Ahopelto, Phys. Rev. Lett. 77, 342 (1996); M. Bayer, O. Stern, A. Kuther, and A. Forchel, Phys. Rev. B 61, 7273 (2000); L.R. Wilson, D.J. Mowbray, M.S. Skolnick, M. Morifuji, M.J. Steer, I.A. Larkin, and M. Hopkinson, ibid. 57, R2073 (1998); P.P. Paskov, P.O. Holtz, B. Monemar, J.M. Garcia, W.V. Schoenfeld, and P.M. Petroff, ibid. 62, 7344 (2000); K.L. Janssens, F.M. Peeters, and V.A. Schweigert, ibid. 63, 205311 (2001); S.-J. Cheng, W. Sheng, and P. Hawrylak, ibid. 68, 235330 (2003); S. Raymond, S. Studenikin, A. Sachrajda, Z. Wasilewski, S.J. Cheng, W. Sheng, P. Hawrylak, A. Babinski, M. Potemski, G. Ortner, and M.
Bayer, Phys. Rev. Lett. 92, 187402 (2004).

${ }^{7}$ N.H. Quang, S. Ohnuma, and A. Natori, Phys. Rev. B 62, 12955 (2000); C. Schulhauser, D. Haft, R.J. Warburton, K. Karrai, A.O. Govorov, A.V. Kalameitsev, A. Chaplik, W. Schoenfeld, J.M. Garcia, and P.M. Petroff, ibid. 66, 193303 (2002); M. Hayne, T. Vanhoucke, and V.V. Moshchalkov, ibid. 68, 035322 (2003).

${ }^{8}$ G. Ortner, M. Bayer, A. Larionov, V.B. Timofeev, A. Forchel, Y.B. Lyanda-Geller, T.L. Reinecke, P. Hawrylak, S. Fafard, and Z. Wasilewski, Phys. Rev. Lett. 90, 086404 (2003); E. Anisimovas and F.M. Peeters, Phys. Rev. B 68, 115310 (2003).

${ }^{9}$ M. Rontani, S. Amaha, K. Muraki, F. Manghi, E. Molinari, S. Tarucha, and D. G. Austing, Phys. Rev. B 69, 085327 (2004).

${ }^{10}$ B. Szafran, B. Stébé, J. Adamowski, and S. Bednarek, Phys. Rev. B 66, 165331 (2002).

${ }^{11}$ D. Loss and D.P. DiVincenzo, Phys. Rev. A 57, 120 (1998).

${ }^{12}$ D. Bellucci, M. Rontani, F. Troiani, G. Goldoni, and E. Molinari, Phys. Rev. B 69, 201308(R) (2004), and references therein.

${ }^{13}$ E.L. Ivchenko and G.E. Pikus, Superlattices and Other Heterostructures (Springer, Berlin, 1997).

${ }^{14}$ M. Bayer, A. Kuther, V.D. Kulakovskii, A. Forchel, P.A. Knipp, and T.L. Reinecke, Phys. Rev. B 64, 201307 (2001).

${ }^{15}$ G. Burkard, G. Seelig, and D. Loss, Phys. Rev. B 62, 2581 (2000).

${ }^{16}$ L.-P. Levy, Magnetism and Superconductivity (Springer, Berlin, 1998).

${ }^{17}$ J. Adamowski, M. Sobkowicz, B. Szafran, and S. Bednarek, 
Phys. Rev. B 62, 4234 (2000).

${ }^{18}$ F. Findeis, M. Baier, A. Zrenner, M. Bichler, G. Abstreiter, U. Hohenester, and E. Molinari, Phys. Rev. B 63, 121309 (2001); A. Hartmann, Y. Ducommun, E. Kapon, U. Hohenester, and E. Molinari, Phys. Rev. Lett. 84, 5648 (2000); E.S. Moskalenko, K.F. Karlsson, P.O. Holtz, B. Monemar, W.V. Schoenfeld, J.M. Garcia, and P.M. Petroff, Phys. Rev. B 64, 085302 (2001); M. Lomascolo, A. Vergine, T.K. Johal, R. Rinaldi, A. Passaseo, R.
Cingolani, S. Patanè, M. Labardi, M. Allegrini, F. Troiani, and E. Molinari, ibid. 66, 041302(R) (2002).

${ }^{19}$ D. Bellucci, F. Troiani, G. Goldoni, and E. Molinari, Physica E (Amsterdam) 22, 482 (2004).

${ }^{20}$ F. Troiani, U. Hohenester, and E. Molinari, Phys. Rev. B 65, 161301(R) (2002); M. Rontani, F. Troiani, U. Hohenester, and E. Molinari, Solid State Commun. 199, 309 (2001). 